\title{
When a crisis hits: bilateral adrenal haemorrhage - a case report
}

\author{
Authors: Fiona Windsor ${ }^{\mathrm{A}}$ and Shvaita Ralhan ${ }^{\mathrm{A}}$
}

\section{Introduction}

We present a case of adrenal insufficiency secondary to bilateral adrenal haemorrhage (BAH) caused by heparin-induced thrombocytopenia (HIT).

\section{Case presentation}

A 76-year-old woman was admitted with a distal femur fracture. This was managed surgically with a gamma nail and she made a good postoperative recovery. Postoperative venous thromboembolism (VTE) prophylaxis was with subcutaneous dalteparin 5,000 units daily. She remained in hospital for ongoing rehabilitation with the aim to discharge her directly home.

On day 5 of her inpatient stay, she developed a urinary tract infection which was managed with oral antibiotics. She then developed non-specific, generalised, severe abdominal and chest pain. Initial clinical examination was unremarkable, as were electrocardiography and chest X-ray. Laboratory parameters were normal including a high-sensitivity troponin. Subsequent surgical review noted that she had raised blood pressure (>200 mmHg systolic). Abdominal computed tomography (CT) revealed a $3 \times 2 \mathrm{~cm}$ left supra/para-renal retroperitoneal mass. Hypertension persisted and she developed a low-grade fever $\left(37.5^{\circ} \mathrm{C}\right)$ associated with confusion and a reduction in consciousness level. The following day she developed delirium and collapsed due to profound postural hypotension. Magnetic resonance imaging (MRI) of the adrenal glands was performed, which showed a new right-sided adrenal mass and an unchanged left-sided mass; concern was raised for adrenal haemorrhage. CT pulmonary angiography demonstrated a small subsegmental pulmonary embolus. CT of her head was normal.

Blood tests showed a platelet count of $53 \times 10^{9} / \mathrm{L}$ (admission count 233), a sodium of $128 \mathrm{mmol} / \mathrm{L}$ (admission $139 \mathrm{mmol} / \mathrm{L}$ ) but a stable haemoglobin and potassium. There were rising inflammatory markers. She was discussed with the haematologists, who requested a D-dimer (raised at 72,514 $\mathrm{ng} / \mathrm{mL}$ ) and fibrinogen (normal at $3.0 \mathrm{~g} / \mathrm{L}$ ). The causes of the new thrombocytopenia were reviewed and the possibility of HIT was raised. Her 4T score, a validated tool for assessing pre-test probability for HIT, ${ }^{1}$ was 7 which confirmed a high possibility for HIT. HIT enzyme-linked

Authors: ${ }^{A}$ Oxford University Hospitals NHS Trust, Oxford, UK immunosorbent assay (ELISA) was positive. Her dalteparin was stopped and she was commenced on subcutaneous fondaparinux. Random serum cortisol was assayed due to the bilateral adrenal changes on CT and marked postural drop. This was low at $32 \mathrm{nmol} / \mathrm{L}$ and a short Synacthen test (SST) showed no adrenal function. She was commenced on hydrocortisone and made a rapid recovery. She was discharged home with endocrine followup. Her drug allergies were updated to include heparin.

Subsequent MRI demonstrated complete resolution of the adrenal haemorrhage, but repeat SST showed no improvement in adrenal function.

\section{Discussion}

HIT is a known cause of thrombocytopenia, but it subsequently causing BAH is less recognised. Case reports demonstrate that common signs and symptoms are non-specific but can include abdominal pain, confusion and low-grade fever. ${ }^{2-5} \mathrm{HIT}$ is more common with unfractionated heparin and following orthopaedic surgery, ${ }^{4,5}$ although it can happen with low molecular weight heparin ${ }^{2,4,5}$ or spontaneously. ${ }^{3}$

HIT can be easily missed and is therefore important to consider in an acutely unwell and deteriorating patient with thrombocytopenia. Furthermore, BAH should feature as a differential diagnosis of thrombotic complications following HIT.

\section{Conflicts of interest statement}

None declared.

\section{References}

1 Watson H, Davidson S, Keeling D. Guidelines on the diagnosis and management of heparin-induced thrombocytopenia: second edition. Br J Haematol 2012;159: 528-40.

2 Saleem N, Khan M, Parveen S, Balavenkatraman A. Bilateral adrenal haemorrhage: a cause of haemodynamic collapse in heparin-induced thrombocytopenia. BMJ Case Rep 2016;2016:bcr2016214679.

3 Warkentin T, Safyan E, Linkins L. Heparin-induced thrombocytopenia presenting as bilateral adrenal haemorrhages. $N$ Engl J Med 2015;372:492-4.

4 Ketha S, Smithedajkul P, Vella A, Pruthi R, Wysokinski W, McBane R. Adrenal haemorrhage due to heparin-induced thrombocytopenia. Thromb Haemost 2013;109:669-75.

5 Rosenberger LH, Smith PW, Sawyer RG, Hanks JB, Adams RB, Hedrick TL. Bilateral adrenal hemorrhage: the unrecognized cause of hemodynamic collapse associated with heparin-induced thrombocytopenia. Crit Care Med 2011;39:833-8. 